\title{
Nonlinear propagation of a wave packet in a hard-walled circular duct
}

\author{
Ali Hasan Nayfeh \\ Department of Engineering Science and Mechanics, Virginia Polytechnic Institute and State University, Blacksburg, Virginia \\ 24061 \\ (Received 30 May 1974; revised 21 November 1974)
}

The method of multiple scales is used to derive a nonlinear Schrödinger equation for the temporal and spatial modulation of the amplitudes and the phases of waves propagating in a hard-walled circular duct. This equation is used to show that monochromatic waves are stable and to determine the amplitude dependance of the cutoff frequencies.

Subject Classification: 25.20; 20.45.

\section{INTRODUCTION}

In many physical problems involving sound transmission in ducts, the sound-pressure levels involved are so high that the problem of propagation and attenuation cannot be treated using the usual linear acoustic analyses. At these high sound-pressure levels, the nonlinear effects play an important role in the attenuation of the sound (see, for example, the survey articles of Blackstock ${ }^{1}$ and Nayfeh et al. ${ }^{2}$ ). These nonlinear effects are of two types-the nonlinearity of the acoustic properties of the lining material and the nonlinearity of the gas itself. In this paper, we consider only the nonlinearity of the gas; that is, we consider waves propagating in hard-walled ducts.

In analyzing waves propagating in a duct, one needs to distinguish between dispersive and nondispersive waves by investigating the linear problem. To this end, we consider linear, inviscid, irrotational waves propagating in a hard-walled duct with a uniform cross sectional area $S^{*}$ and filled.with a fluid having a uniform pressure $p_{0}^{*}$ and a uniform density $\rho_{0}^{*}$. We introduce a Cartesian coordinate system such that the $x$ axis is along the axis of the duct. Moreover, we introduce dimensionless quantities by using a characteristic length $R^{*}$ of the cross section, the ambient speed of sound $c_{0}^{*}$, and the ambient density $\rho_{0}^{*}$ as reference quantities. Thus, we let $\mathbf{r}^{*}=\mathbf{r}^{*} / R^{*}, \nabla=\nabla^{*} / c_{0}^{*}, \rho=\rho^{*} / \rho_{0}^{*}, p=p^{*} /$ $\rho_{0}^{*} c_{0}^{* 2}$, and $t=t^{*} c_{0}^{*} / R^{*}$, where starred and unstarred quantities denote dimensional and dimensionless quantities, respectively, $\mathbf{r}$ is the position vector, $\nabla$ is the velocity vector, $\rho$ is the gas density, $p$ is the gas pressure, and $t$ is the time. In terms of these dimensionless quantities, the dimensionless velocity potential $\phi(r, t)$ is governed by

$$
\begin{aligned}
& \nabla^{2} \phi-\phi_{t t}=0, \\
& \partial \phi / \partial n=0, \text { at } C,
\end{aligned}
$$

where $C$ is the equation of the duct wall and $n$ is the normal to the wall.

For a sinusoidally varying wave, we let

$$
\phi(x, y, z, t)=\Psi(y, z) \exp [i(k x-\omega t)] .
$$

Substituting Eq. 3 into Eqs. 1 and 2, we obtain

$$
\Psi_{y y}+\Psi_{z x}+\kappa^{2} \Psi=0,
$$

$$
8 \Psi / \partial n=0 \text {, at } C,
$$

where $\omega$ and $k$ satisfy the dispersion relationship

$$
\omega^{2}-k^{2}=\kappa^{2} \text {. }
$$

The waves are called nondispersive if $\kappa=0$ because all waves irrespective of their wavenumbers travel with the same phase speed $\omega / k$; otherwise, they are dispersive. Thus, nondispersive waves correspond to $\Psi=$ const from Eqs. 4 and 5; that is, they correspond to plane waves. Including the tube-wall effects (acoustic boundary layer) leads to almost plane waves (i.e., $\kappa \neq 0$, but small) which are weakly dispersive. ${ }^{3.4}$ Since all linear waves travel with exactly or approximately the same phase speed, one cannot excite a single frequency wave (a monochromatic wave) without strongly exciting all its harmonics. In this case, the effect of the gas nonlinearity leads to a cumulative distortion of the wave owing to the increase of the phase speed with increasing amplitude. This distortion results in a shift of energy among the several harmonics and requires that the waveform be analyzed directly from the nonlinear equations of motion.

For dispersive waves, $n$th harmonic resonance exists whenever $(\omega, k)$ and $(n \omega, n k)$, where $n$ is an integer, simultaneously satisfy the dispersion relationship, Eq. 6. In this case, one cannot excite one of these waves without strongly exciting its resonant wave. For twodimensional ducts, $\kappa=n \pi$ if $R^{*}$ is taken to be the halfwidth of the duct. Thus, every wave is resonant with all its harmonics, and one cannot determine a uniform expansion by treating a monochromatic wave without considering its interaction with all its harmonics. For rectangular ducts, $\kappa^{2}=n^{2} \pi^{2} / b_{1}^{2}+m^{2} \pi^{2} / b_{2}^{2}$, where $2 b_{1}$ and $2 b_{2}$ are the dimensions of the cross section. Thus, any wave is resonant with all its harmonics (one needs simply to set $m=n$ ). In this case also, one cannot determine a uniform expansion by treating a monochromatic wave without considering its interaction with all its harmonics. In what follows, we exclude the case of harmonic resonance. Although our method of analysis is valid for all nondispersive waves without harmonic resonance, we treat the case of a circular cross section so that we would be able to give an explicit solution.

To the author's knowledge (see, Nayfeh et al. ${ }^{2}$ ), all linear and nonlinear analysis of strongly dispersive 
waves in a duct deal with a monochromatic wave (a single frequency sound). Thus, none of the existing analysis can treat the case of the propagation of a sound consisting of a group of waves centered about a frequency $\omega$. In particular, they cannot treat the simple problem of the propagation of two waves of frequencies $\omega$ and $\omega+\Delta \omega$ produced by a piston; the initial condition in this case can be written as

$$
u=a_{1} \cos \omega t+a_{2} \cos [(\omega+\Delta \omega) t+\tilde{\theta}],
$$

where $u$ is the velocity of the piston, $a_{1}$ and $a_{2}$ are the initial amplitudes of the two produced waves, and $\tilde{\theta}$ is the phase difference. Expanding the second trigonometric function in Eq. 7, we rew rite it as

$$
u=a_{0}(t) \cos \left[\omega t+\theta_{0}(t)\right],
$$

where

$$
\begin{aligned}
& a_{0}^{2}=\left\{a_{1}+a_{2} \cos [(\Delta \omega) t+\tilde{\theta}]\right\}^{2}+a_{2}^{2} \sin ^{2}[(\Delta \omega) t+\tilde{\theta}], \\
& \tan \theta=\left\{a_{2} \sin [(\Delta \omega) t+\tilde{\theta}]\right\} /\left\{a_{1}+a_{2} \cos [(\Delta \omega) t+\tilde{\theta}]\right\} .
\end{aligned}
$$

Thus, when $\Delta \omega$ is small, the initial condition consists of a wave with a frequency $\omega$ whose amplitude and phase vary slowly with time. The initial conditions corresponding to a piston executing a motion consisting of a small band of frequencies centered at $\omega$ can also be expressed as in Eq. 8. As the wave propagates down the duct, the amplitude and the phase of the wave will also modulate with the axial distance. The purpose of the present paper is to derive partial differential equations describing these modulations and to use these equations to determine the stability of monochromatic waves and the dependence of the cutoff frequency on the amplitude.

\section{PROBLEM FORMULATION}

We consider finite-amplitude waves propagating in a hard-walled cylindrical duct. The fluid is assumed to be inviscid, irrotational, and initially quiescent with a uniform pressure $p_{0}^{*}$ and a uniform density $\rho_{0}^{*}$ so that its subsequent motion can be reprèsented by a potential function. Boundary-layer effects are incorporated in Sec. III.

We introduce a cylindrical coordinate system $(r, \theta, x)$ whose $x$ axis coincides with the duct axis. We introduce dimensionless quantities as in the previous section and choose $R^{*}$ to be the radius of the duct. In terms of these dimensionless variables, the equations describing the conservation of mass and momentum are

$$
\begin{aligned}
& \frac{\partial \rho}{\partial t}+\nabla \cdot(\rho \nabla)=0, \\
& \rho\left(\frac{\partial \mathrm{v}}{\partial t}+\nabla \cdot \nabla v\right)=-\nabla p .
\end{aligned}
$$

The pressure is related to the density by the isentropic relationship

$$
p^{*} / p_{0}^{*}=\left(\rho^{*} / \rho_{0}^{*}\right)^{\gamma}
$$

or in dimensionless quantities by

$$
\gamma p=\rho^{\gamma},
$$

where $\gamma$ is the gas specific heat ratio. Since the duct walls are assumed to be rigid, the appropriate boundary condition is the vanishing of the normal velocity at the duct walls; that is,

$$
v=0 \text { at } r=1 \text {, }
$$

where $v$ is the radial component of velocity.

Since the flow is assumed to be inviscid and irrotational, the velocity $\boldsymbol{v}$ is derivable from a potential function $\phi(\mathbf{r}, t)$ according to

$$
\nabla=\nabla \phi \text {. }
$$

Substituting for $p$ and $\nabla$ from Eqs. 12 and 14 into Eq. 11, using the irrotationality of the gas, and integrating the resulting equation, we obtain

$$
\rho^{\gamma-1}=1+(1-\gamma)\left[\phi_{t}+(1 / 2)(\nabla \phi)^{2}\right] .
$$

Eliminating $\rho$ from Eqs. 12 and 15 gives

$$
\gamma p=\left\{1+(1-\gamma)\left[\phi_{t}+(1 / 2)(\nabla \phi)^{2}\right]\right]^{\gamma /(\gamma-1)} .
$$

Differentiating Eq. 15 with respect to $t$, eliminating $\rho$ by using Eqs. 10, 14, and 15, and arranging, we obtain

$$
\begin{aligned}
\phi_{t t}-\nabla^{2} \phi= & (1-\gamma)\left[\phi_{t}+(1 / 2)(\nabla \phi)^{2}\right] \nabla^{2} \phi \\
& -\frac{\partial}{\partial t}(\nabla \phi)^{2}-(1 / 2) \nabla \phi \cdot \nabla(\nabla \phi)^{2} .
\end{aligned}
$$

In terms of the potential function $\phi$, the boundary condition 13 becomes

$$
\phi_{r}=0 \text {, at } r=1 \text {. }
$$

To determine an approximate solution to Eq. $17 \mathrm{sub}-$ ject to the boundary condition, Eq. 18, allowing for waves modulating with both space and time, we use the method of multiple scales (e.g., Chap. 6 of Ref. 5) and let

$$
\phi(r, \theta, x, t)=\sum_{n=1}^{3} \epsilon^{n} \phi_{n}\left(r, \theta, X_{0}, X_{1}, X_{2}, T_{0}, T_{1}, T_{2}\right)+0\left(\epsilon^{4}\right),
$$

where $\epsilon$ is a small but finite dimensionless parameter characterizing the amplitude of the wave and

$$
X_{n}=\epsilon^{n} x, T_{n}=\epsilon^{n} t \text {. }
$$

Here, $X_{0}$ is a short scale characterizing the wavelength, $X_{1}$ and $X_{2}$ are long scales characterizing the amplitude and phase modulations with axial distance, $T_{0}$ is a short scale characterizing the frequency of the wave, and $T_{1}$ and $T_{2}$ are long scales characterizing the temporal amplitude and phase modulations. Using Eq. 20 and the chain rule, we express the temporal and axial derivatives as

$$
\begin{aligned}
& \frac{\partial}{\partial x}=\frac{\partial}{\partial X_{0}}+\epsilon \frac{\partial}{\partial X_{1}}+\epsilon^{2} \frac{\partial}{\partial X_{2}}+\cdots, \\
& \frac{\partial}{\partial t}=\frac{\theta}{\partial T_{0}}+\epsilon \frac{\partial}{\partial T_{1}}+\epsilon^{2} \frac{\partial}{\partial T_{2}}+\cdots .
\end{aligned}
$$

Substituting Eqs. 19-21 into Eqs. 17 and 18 and equating coefficients of like powers of $\epsilon$, we obtain:

$$
\begin{aligned}
& \text { Order } \epsilon- \\
& \qquad \mathcal{L}\left(\phi_{1}\right)=\left(\frac{\partial^{2}}{\partial T_{0}^{2}}-\nabla_{0}^{2}\right) \phi_{1}=0,
\end{aligned}
$$




$$
\begin{aligned}
& \nabla_{0}^{2} \equiv \frac{\partial^{2}}{\partial r^{2}}+\frac{1}{r} \frac{\partial}{\partial r}+\frac{1}{r^{2}} \frac{\partial^{2}}{\partial \theta^{2}}+\frac{\partial^{2}}{\partial X_{0}^{2}}, \\
& \partial \phi_{1} / \partial r=0, \text { at } r=1 ;
\end{aligned}
$$

order $\epsilon^{2}-$

$$
\begin{aligned}
& \mathscr{L}\left(\phi_{2}\right)=-2 \frac{\partial^{2} \phi_{1}}{\partial T_{0} \partial T_{1}}+2 \frac{\partial^{2} \phi_{1}}{\partial X_{0} \partial X_{1}} \\
&+(1-\gamma) \frac{\partial \phi_{1}}{\partial T_{0}} \nabla_{0}^{2} \phi_{1}-\frac{\partial}{\partial T_{0}}\left(\nabla_{0} \phi_{1}\right)^{2}, \\
& \partial \phi_{2} / \partial r=0, \quad \text { at } r=1 ;
\end{aligned}
$$

Order $\epsilon^{3}-$

$$
\begin{aligned}
\mathcal{L}\left(\phi_{3}\right)= & -2 \frac{\partial^{2} \phi_{1}}{\partial T_{0} \partial T_{2}}-\frac{\partial^{2} \phi_{1}}{\partial T_{1}^{2}}-2 \frac{\partial^{2} \phi_{2}}{\partial T_{0} \partial T_{1}}+2 \frac{\partial^{2} \phi_{1}}{\partial X_{0} \partial X_{2}} \\
& +\frac{\partial^{2} \phi_{1}}{\partial X_{1}^{2}}+2 \frac{\partial^{2} \phi_{2}}{\partial X_{0} \partial X_{1}}+(1-\gamma) \frac{\partial \phi_{1}}{\partial T_{1}} \nabla_{0}^{2} \phi_{1} \\
& +2(1-\gamma) \frac{\partial \phi_{1}}{\partial T_{0}} \frac{\partial^{2} \phi_{1}}{\partial X_{0} \partial X_{1}}+(1-\gamma) \frac{\partial \phi_{1}}{\partial T_{0}} \nabla_{0}^{2} \phi_{2} \\
& +(1-\gamma) \frac{\partial \phi_{2}}{\partial T_{0}} \nabla_{0}^{2} \phi_{1}+\frac{1}{2}(1-\gamma)\left(\nabla_{0} \phi_{1}\right)^{2} \nabla_{0}^{2} \phi_{1}-\frac{\partial}{\partial T_{1}}\left(\nabla_{0} \phi_{1}\right)^{2} \\
& -2 \frac{\partial}{\partial T_{0}}\left(\frac{\partial \phi_{1}}{\partial X_{0}} \frac{\partial \phi_{1}}{\partial X_{1}}\right)-2 \frac{\partial}{\partial T_{0}}\left(\nabla_{0} \phi_{1} \cdot \nabla_{0} \phi_{2}\right) \\
& -\frac{1}{2} \nabla_{0} \phi_{1} \cdot \nabla_{0}\left(\nabla_{0} \phi_{1}\right)^{2}, \\
\partial \phi_{3} / \partial r & =0, \text { at } r=1
\end{aligned}
$$

\section{SOLUTION}

We take the solution of Eqs. 22 that is bounded at the axis in the form of a traveling wave packet centered at the frequency $\omega$ and the wavenumber $k$; that is, we let

$$
\begin{aligned}
& \phi_{1}=A\left(X_{1}, X_{2}, T_{1}, T_{2}\right) J_{m}(\kappa r) \exp (i \zeta)+c c, \\
& \zeta=k X_{0}-\omega T_{0}+m \theta,
\end{aligned}
$$

where $J_{m}$ is Bessel's function of order $m$, cc stands for the complex conjugate of the preceding terms, and $\omega$ and $k$ satisfy the dispersion relationship

$$
\omega^{2}-k^{2}=\kappa^{2} \text {. }
$$

We note that Eqs. 28 describe a single frequency wave only when $A$ is independent of either $X_{1}$ and $X_{2}$ or $T_{1}$ and
$T_{2}$. For nondispersive waves, one must include all the harmonics in Eq. 28a. Substituting Eqs. 28 into Eq. 23, we have

$$
J_{m}^{\prime}(\kappa)=0 \text {. }
$$

In what follows, we exclude the nondispersive case corresponding to $\kappa=0$ (i.e., plane waves). Note that the function $A$ is still undetermined at this level of approximation; it is determined by invoking the so-called solvability condition in the second- and third-order problems. tain

Substituting for $\phi_{1}$ from Eq. 28a into Eq. 24, we ob-

$$
\begin{aligned}
\mathscr{L}\left(\phi_{2}\right)= & 2 i\left(\omega \frac{\partial A}{\partial T_{1}}+k \frac{\partial A}{\partial X_{1}}\right) J_{m}(\kappa r) \exp (i \zeta) \\
& +\left[2 i \omega \kappa^{2} J_{m+1}^{2}(\kappa r)-4 i \omega \kappa m \frac{1}{r} J_{m+1}(\kappa r) J_{m}(\kappa r)\right. \\
& \left.-i \omega\left(\gamma \omega^{2}-\kappa^{2}+k^{2}\right) J_{m}^{2}(\kappa r)\right] A^{2} \exp (2 i \zeta)+\mathrm{cc}
\end{aligned}
$$

Since the homogeneous second-order problem consisting of Eqs. 25 and 31 is the same as the first-order problem and since the latter has a nontrivial solution, the inhomogeneous second-order problem has a solution if, and only if, a solvability condition is satisfied. This condition is equivalent to the elimination of secular terms. To determine this solvability condition, we seek a particular solution of the form

$$
\phi_{2}=\Phi\left(r, X_{1}, X_{2}, T_{1}, T_{2}\right) \exp (i \zeta) .
$$

Substituting this solution into Eqs. 31 and 25 and equating the coefficients of $\exp (i \zeta)$ on both sides, we have

$\frac{\partial^{2} \Phi}{\partial r^{2}}+\frac{1}{r} \frac{\partial \Phi}{\partial r}+\left(\kappa^{2}-\frac{m^{2}}{r^{2}}\right) \Phi=2 i\left(\omega \frac{\partial A}{\partial T_{1}}+k \frac{\partial A}{\partial X_{1}}\right) J_{m}(\kappa r)$,

$\partial \Phi / \partial r=0, \quad$ at $r=1$.

Determining the solvability condition of Eqs. 25 and 31 is transformed into that of determining the solvability condition of Eqs. 33 and 34. To this end, we multiply Eq. 33 by $r \Phi^{*}\left(r, X_{1}, T_{1}\right)$, where $\Phi^{*}$ is specified later, integrate the resulting equation by parts from $r=0$ to $r=1$, and obtain

$$
\int_{0}^{1} \Phi\left[\frac{\partial}{\partial r}\left(r \frac{\partial \Phi^{*}}{\partial r}\right)+\left(\kappa^{2} r-\frac{m^{2}}{r}\right) \Phi^{*}\right] d r+\left[r \Phi^{*} \frac{\partial \Phi}{\partial r}-r \Phi \frac{\partial \Phi *}{\partial r}\right]_{r=0}^{r=1}=2 i\left(\omega \frac{\partial A}{\partial T_{1}}+k \frac{\partial A}{\partial X_{1}}\right) \int_{0}^{1} r \Phi^{*} J_{m}(\kappa r) d r
$$

W e choose $\Phi^{*}$ to be a solution of the so-called adjoint homogeneous problem; that is,

$$
\begin{aligned}
& \frac{\partial}{\partial r}\left(r \frac{\partial \Phi^{*}}{\partial r}\right)+\left(\kappa^{2} r-\frac{m^{2}}{r}\right) \Phi^{*}=0, \\
& \partial \Phi * / \partial r=0, \text { at } r=1 .
\end{aligned}
$$

A solution of Eqs. 36 and 37 that is bounded at the origin is $\Phi *=J_{m}(\kappa r)$. Substituting for $\Phi *$ into Eq. 35 and using Eqs. 34 and 37, we find that the solvability condition is

$$
\omega \frac{\partial A}{\partial T_{1}}+k \frac{\partial A}{\partial X_{1}}=0
$$

With this solvability condition, the solution of the second-order problem is

$$
\begin{aligned}
\phi_{2}= & {\left[\Gamma_{1} J_{m}^{2}(\kappa r)+\Gamma_{2} r J_{m}(\kappa r) J_{m+1}(\kappa r)+\Gamma_{3} J_{2 m}(2 \kappa r)\right] A^{2} } \\
& \times \exp (2 i \zeta)+c c,
\end{aligned}
$$$$
\text { where }
$$

$\Gamma_{1}=-i \omega\left[(1 / 2) m(\gamma+1) \omega^{2} \kappa^{-2}+1\right], \quad \Gamma_{2}=(1 / 2) i(\gamma+1) \omega^{3} \kappa^{-1}$, $\Gamma_{3}=-(1 / 2) \Gamma_{2} J_{m}(\kappa)\left[J_{m+1}(\kappa)+J_{m+1}^{\prime}(\kappa)\right] / \kappa J_{m}^{\prime}(2 \kappa)$. 
Note that the $\Gamma_{i} \rightarrow \infty$ and hence $\phi_{2} \rightarrow \infty$ as $\kappa \rightarrow 0$ (i. e., plane waves); otherwise $\phi_{2}$ is bounded for all other $\kappa$. This is the reason we excluded the nondispersive case because in that case one cannot take the first-order solution to consist of a small band of waves centered about the frequency $\omega$ as in Eqs. 28. Equation 38 shows that the function $A$ describing the amplitude and phase modulations of the wave is linear. Hence, we need to carry out the expansion to at least one more order to determine the nonlinear effects.

Substituting for $\phi_{1}$ and $\phi_{2}$ from Eqs. 28 and 39 into Eq. 26, we have

$$
\begin{aligned}
\mathscr{L}\left(\phi_{3}\right)=\left[2 i\left(\omega \frac{\partial A}{\partial T_{2}}+k \frac{\partial A}{\partial X_{2}}\right)-\frac{\partial^{2} A}{\partial T_{1}^{2}}+\frac{\partial^{2} A}{\partial X_{1}^{2}}\right] J_{m}(\kappa r) \exp (i \zeta) \\
-A^{2} \bar{A} F(r) \exp (i \zeta)+c c \\
+ \text { harmonics other than } \exp ( \pm i \zeta),
\end{aligned}
$$

where $F(r)$ is given in Appendix A. Since the homogeneous third-order problem consisting of Eqs. 27 and 40 has a nontrivial solution, the corresponding inhomogeneous problem has a solution if, and only if, a solvability condition is satisfied. To determine this solvability condition, we seek a particular solution of the form

$$
\phi_{3}=\psi\left(r, X_{1}, X_{2}, T_{1}, T_{2}\right) \exp (i \zeta) .
$$

Substituting this solution into Eqs. 27 and 40 and equating the coefficients of $\exp (i \zeta)$ on both sides, we obtain

$$
\begin{aligned}
\frac{\partial^{2} \psi}{\partial r^{2}} & +\frac{1}{r} \frac{\partial \psi}{\partial r}+\left(\kappa^{2}-\frac{m^{2}}{r^{2}}\right) \psi \\
& =\left[2 i\left(\omega \frac{\partial A}{\partial T_{2}}+k \frac{\partial A}{\partial X_{2}}\right)-\frac{\partial^{2} A}{\partial T_{1}^{2}}+\frac{\partial^{2} A}{\partial X_{1}^{2}}\right] J_{m}(\kappa r)-A^{2} \bar{A} F(r),
\end{aligned}
$$

$$
\partial \psi / \partial r=0, \quad \text { at } r=1 \text {. }
$$

The solvability condition of Eqs. 42 and 43 can be obtained in a manner similar to that used to determine the solvability condition of Eqs. 33 and 34. Multiplying Eq. 42 by $r J_{m}(\kappa r)$, integrating by parts from $r=0$ to $r=1$, and using Eq. 43, we obtain the solvability condition in the form

$$
2 i\left(\omega \frac{\partial A}{\partial T_{2}}+k \frac{\partial A}{\partial X_{2}}\right)-\frac{\partial^{2} A}{\partial T_{1}^{2}}+\frac{\partial^{2} A}{\partial X_{1}^{2}}=\Lambda A^{2} \bar{A},
$$

where

$$
\Lambda=\left[\int_{0}^{1} r F(r) J_{m}(\kappa r) d r\right] /\left[\int_{0}^{1} r J_{m}^{2}(\kappa r) d r\right] .
$$

Eliminating $\partial^{2} A / \partial X_{1}^{2}$ from Eq. 44 by using Eq. 38 gives

$$
2 i\left(\omega \frac{\partial A}{\partial T_{2}}+k \frac{\partial A}{\partial X_{2}}\right)+\left(\frac{\omega^{2}}{k^{2}}-1\right) \frac{\partial^{2} A}{\partial T_{1}^{2}}=\Lambda A^{2} \bar{A} .
$$

To simplify Eq. 46, we differentiate the dispersion relationship 29 with respect to $\omega$ and obtain

$$
k k^{\prime}=\omega,
$$

where $k^{\prime}=d k / d \omega$ the inverse of the group velocity. Differentiating Eq. 47 with respect to $\omega$ gives

$$
k k^{\prime \prime}=1-k^{\prime 2}=1-\omega^{2} / k^{2} \text {. }
$$

Using Eqs. 47 and 48, letting $T_{n}=\epsilon^{n} t$ and $X_{2}=\epsilon^{2} x$, and arranging, we rewrite Eq. 46 as

$$
\frac{\partial A}{\partial x}+k^{\prime} \frac{\partial A}{\partial t}+\frac{1}{2} i k^{\prime \prime} \frac{\partial^{2} A}{\partial t^{2}}=\frac{1}{2} i \epsilon^{2} \frac{\Lambda}{k} A^{2} \bar{A} .
$$

Changing the independent variables from $x$ and $t$ to

$$
\xi=t-k^{\prime} x, \quad \eta=x,
$$

we express Eq. 49 in the form

$$
\frac{\partial A}{\partial \eta}+\frac{1}{2} i k^{\prime \prime} \frac{\partial^{2} A}{\partial \xi^{2}}=-\frac{1}{2} i \epsilon^{2} \frac{\Lambda}{k} A^{2} \bar{A}
$$

which is a nonlinear Schrődinger equation. ${ }^{6}$ Letting $A$ $=\frac{1}{2} a \exp (i \beta)$ with real $a$ and $\beta$ in Eq. 51 and separating real and imaginary parts, we obtain

$$
\begin{aligned}
& \frac{\partial a}{\partial \eta}-k^{\prime \prime}\left[\frac{\partial a}{\partial \xi} \frac{\partial \beta}{\partial \xi}+\frac{1}{2} a \frac{\partial^{2} \beta}{\partial \xi^{2}}\right]=0, \\
& \frac{\partial \beta}{\partial \eta}+\frac{1}{2} k^{\prime \prime}\left[\frac{1}{a} \frac{\partial^{2} a}{\partial \xi^{2}}-\left(\frac{\partial \beta}{\partial \xi}\right)^{2}\right]=-\frac{1}{8} \epsilon^{2} \frac{\Lambda}{k} a^{2} .
\end{aligned}
$$

\section{A. The case of monochromatic waves}

For monochromatic waves (a single frequency wave), the amplitude and phase are independent of $t$ so that $A$ of Eqs. 28 is a function of $X_{1}$ and $X_{2}$ only; that is, $\partial a / \partial \xi$ $=\partial \beta / \partial \xi=0$, and Eqs. 52 and 53 can be integrated to give

$$
a=a_{0} \text { and } \beta=-(1 / 8) \epsilon^{2} \frac{\Lambda}{k} a_{0}^{2} \eta+\beta_{0} \text {, }
$$

where $a_{0}$ and $\beta_{0}$ are constants. Substituting for $a$ and $\beta$ from Eqs. 54 into Eqs. 28 and 39, we rewrite Eq. 19 in terms of the original variables as

$$
\begin{aligned}
\phi= & \epsilon a_{0} J_{m}(\kappa r) \cos \left(\hat{k} x-\omega t+m \theta+\beta_{0}\right) \\
& +(1 / 2) \epsilon^{2} a_{0}^{2}\left[\Gamma_{1} J_{m}^{2}(\kappa r)+\Gamma_{2} r J_{m}(\kappa r) J_{m+1}(\kappa r)+\Gamma_{3} J_{2 m}(2 \kappa r)\right] \\
& \times \cos \left[2\left(\hat{k} x-\omega t+m \theta+\beta_{0}\right)\right]+0\left(\epsilon^{3}\right),
\end{aligned}
$$

where

$$
\hat{k}=k-(1 / 8) \epsilon^{2} \Lambda k^{-1} a_{0}^{2} .
$$

This result can be obtained as a special case from the solution of Nayfeh and Tsai ${ }^{7}$ by letting the resistivity of the acoustic material be infinite. Moreover, our solution reduces when $m=0$ (i.e., symmetric modes) to that of Keller and Millman, ${ }^{8}$ which they obtained using the method of strained parameters. In the latter two analysis, the stability of monochromatic waves cannot be investigated. We note that the perturbation parameter $\epsilon$ occurs always as a multiplicative of $a_{0}$, whose value does not affect the solution provided that one interprets $\epsilon a_{0}$ as the initial amplitude of the wave. Hence, $\epsilon$ is just a perturbation parameter.

Equation 56 shows that the nonlinearity of the gas results in a shift in the wavenumber from $k$ to $\hat{k}=k-(1)$ 8) $\epsilon^{2} \Lambda k^{-1} a^{2}$. The variation of $(1 / 8) \Lambda \kappa^{-1}$ with $\kappa$ is shown in Figs. 1 and 2 for eight modes. Since $\Lambda$ is positive, the nonlinearity shifts the wavenumber to lower values resulting in higher phase speeds. The wavenumber shift increases with increasing frequency and decreases with increasing azimuthal mode number.

Equations 52 and 53 can be used to analyze the stabili- 
ty of the aforementioned monochromatic solution. To do this, we let

$$
a=a_{0}+a_{1}, \quad \beta=-(1 / 8) \epsilon^{2} \frac{\Lambda}{k} a_{0}^{2} \eta+\beta_{0}+\beta_{1},
$$

where $a_{1}$ and $\beta_{1}$ are small compared with the preceding terms. Substituting Eq. 57 into Eqs. 52 and 53 and neglecting the nonlinear terms in $a_{1}$ and $\beta_{1}$, we obtain

$$
\begin{aligned}
& \frac{\partial a_{1}}{\partial \eta}-\frac{1}{2} k^{\prime \prime} a_{0} \frac{\partial^{2} \beta_{1}}{\partial \xi^{2}}=0, \\
& \frac{\partial \beta_{1}}{\partial \eta}+\frac{1}{2} k^{\prime \prime} \frac{1}{a_{0}} \frac{\partial^{2} a_{1}}{\partial \xi^{2}}=-\frac{1}{4} \epsilon^{2} \frac{\Lambda}{k} a_{0} a_{1} .
\end{aligned}
$$

Since Eqs. 58 and 59 linear, we seek their solution in the form

$a_{1}=\tilde{a}_{1} \exp [i(\tilde{k} \eta-\tilde{\omega} \xi)], \quad \beta_{1}=\tilde{\beta}_{1} \exp [i(\tilde{k} \eta-\tilde{\omega} \xi)]$,

where $\tilde{a}_{1}$ and $\beta_{1}$ are constants. Substituting this solution into Eqs. 58 and 59 and eliminating $\tilde{a}_{1}$ and $\tilde{\beta}_{1}$, we obtain

$$
\tilde{k}^{2}=\frac{1}{4} k^{\prime \prime} \tilde{\omega}^{2}\left(\tilde{\omega}^{2}-\frac{1}{2} \epsilon^{2} \Lambda a_{0}^{2} / k k^{\prime \prime}\right),
$$

which shows that, if $\Lambda / k^{n}<0, \tilde{k}$ is always real for all values of $\tilde{\omega}$ so that the monochromatic waves given by Eqs. 55 and 56 are neutrally stable. On the other hand, if $\Lambda / k^{\prime \prime}>0, \tilde{k}^{2}$ is negative for all $\bar{\omega}<\epsilon a_{0}\left(\Lambda / 2 k k^{\prime \prime}\right)^{1 / 2}$; consequently, disturbances grow exponentially with $\xi$ and monochromatic waves are unstable. Since Figs. 1 and 2 show that $(1 / 8) \Lambda k^{-1}$ is positive, monochromatic waves are stable only if $k^{\prime \prime}<0$. However, Eqs. 29 and 48 show that $k^{\prime \prime}=-\kappa^{2} / k^{3}<0$. Therefore, monochromatic waves are stable.

\section{B. Solution near cutoff frequencies}

Although Eqs. 55 and 56 are valid for a wide range of frequencies, they break down as $k \rightarrow 0$ (i.e., near the

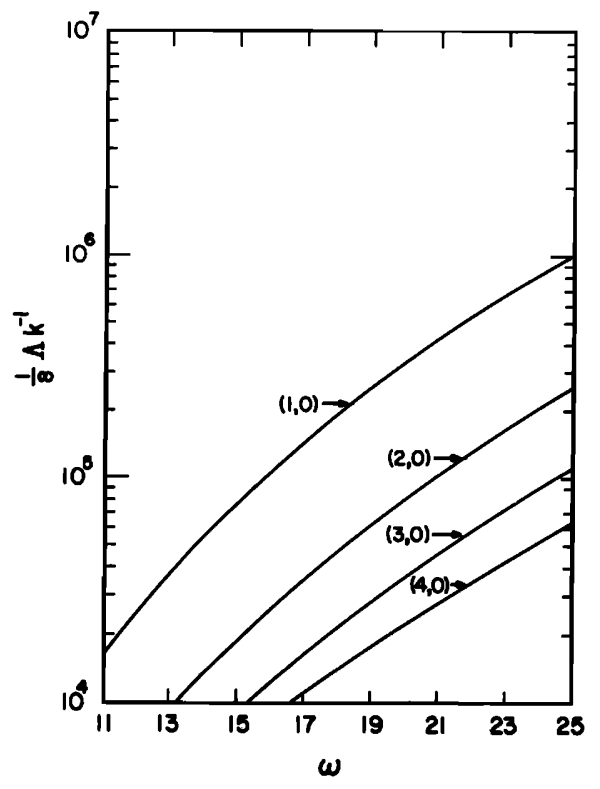

FIG. 1. Variation of the nonlinear wavenumber shift with frequency in a hard-walled duct for four radial modes corresponding to the lowest azimuthal mode.

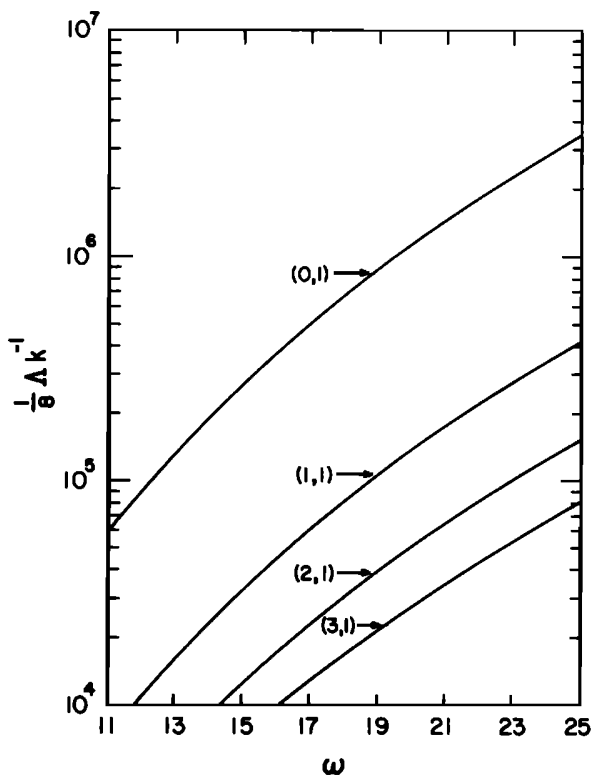

FIG. 2. Variation of the nonlinear wavenumber shift with frequency in a hard-walled duct for the lowest four radial modes corresponding to the first azimuthal mode.

linear cutoff frequencies) because the wavenumber shift approaches infinity. However, the basic equations (Eqs. 38 and 44) for the modulation of the amplitude and the phase with axial distance and time are valid for all frequencies. In this section, we specialize these equations to frequencies near the cutoff values. To do this, we use Eq. 38 to express $\partial A / \partial T_{1}$ as $-(k / \omega) \partial A / \partial X_{1}$, substitute this result into $\mathrm{Eq}$. 44 , and obtain

$$
2 i \omega \frac{\partial A}{\partial T_{2}}+2 i k \frac{\partial A}{\partial X_{2}}+\left(1-\frac{k^{2}}{\omega^{2}}\right) \frac{\partial^{2} A}{\partial X_{1}^{2}}=\Lambda A^{2} \bar{A} .
$$

Letting $T_{2}=\epsilon^{2} t$ and $X_{n}=\epsilon^{n} x$ in Eq. 62, we rewrite it as

$$
\begin{aligned}
& 2 i \omega \frac{\partial A}{\partial t}+2 i k \frac{\partial A}{\partial x}+\left(1-\frac{k^{2}}{\omega^{2}}\right) \frac{\partial^{2} A}{\partial x^{2}}=\epsilon^{2} \Lambda A^{2} \bar{A}, \\
& \frac{\partial A}{\partial t}+\omega^{\prime} \frac{\partial A}{\partial x}-\frac{1}{2} i \omega^{\prime \prime} \frac{\partial^{2} A}{\partial x^{2}}=-\frac{1}{2} i \Lambda \omega^{-1} A^{2} \bar{A} .
\end{aligned}
$$

For monochromatic waves, $\partial A / \partial t=0$ and Eq. 63a becomes

$$
2 i k \frac{d A}{d x}+\left(1-\frac{k^{2}}{\omega^{2}}\right) \frac{d^{2} A}{d x^{2}}=\epsilon^{2} \Lambda A^{2} \bar{A},
$$

which is valid for all frequencies away from zero.

Equation 64 has solutions of the form

$$
A=(1 / 2) a \exp (i \beta),
$$

where $a$ is constant and

$$
\frac{d \beta}{d x}=\left\{-k+\left[k^{2}-\frac{1}{4} \epsilon^{2}\left(1-\frac{k^{2}}{\omega^{2}}\right) \Lambda a^{2}\right]^{1 / 2}\right\}\left(1-\frac{k^{2}}{\omega^{2}}\right)^{-1} .
$$

Away from the cutoff frequencies, $k$ is away from zero and the radical in Eq. 66 can be expanded for small $\epsilon$, yielding

$$
\frac{d \beta}{d x}=-\frac{1}{8} \epsilon^{2} \Lambda k^{-1} a^{2}
$$


in agreement with the monochromatic solution obtained above. On the other hand, when $k \rightarrow 0$ (i.e., near the cutoff frequencies), Eq. 66 tends to

$$
\frac{d \beta}{d x}=-k+\left(k^{2}-\frac{1}{4} \epsilon^{2} \Lambda a^{2}\right)^{1 / 2} .
$$

Substituting for $A$ from Eq. 65 into Eqs. 28 and 39 , using Eq. 68 and letting $T_{0}=t$ and $X_{n}=\epsilon^{\eta} x$, we obtain Eq. 55; however, $\bar{k}$ of Eq. 56 is modified to

$$
k=\left(k^{2}-\frac{1}{4} \epsilon^{2} \Lambda a^{2}\right)^{1 / 2} \text {. }
$$

Therefore, the cutoff frequencies are solutions of

$$
k^{2}-(1 / 4) \epsilon^{2} \Lambda a^{2}=0 \text {. }
$$

Since $k^{2}=\omega^{2}-\kappa^{2}$ according to Eq. 29, the cutoff frequencies are

$$
\omega=\kappa+(1 / 8) \epsilon^{2} a^{2} \Lambda(\kappa)+\cdots,
$$

where $\Lambda(\kappa)$ stands for the value of $\Lambda$ when $\omega=\kappa$. These cutoff frequencies reduce when $m=0$ to those obtained by Keller. ${ }^{9}$

Using Eq. 63a, one can carry out a stability analysis and show that this modified solution is stable.

\section{BOUNDARY-LAYER EFFECTS}

Although viscous effects can be neglected everywhere in the body of the fluid for strongly dispersive waves owing to the absence of shock waves (in contrast with plane, or almost plane, waves which distort over distances that depend on the initial amplitude and frequency of the wave to form shock waves), they cannot be neglected near the duct walls (i.e., the acoustic boundary layer). For example, for waves in circular ducts, the usual linear theory yields ${ }^{2}$

$$
\phi(r, \theta, x, t)=\hat{A}_{n} J_{n}(\hat{\kappa} r) \exp [i(\hat{k} x-\omega t+n \theta)]+c c,
$$

where

$$
\hat{\kappa}^{2}=\omega^{2}-\hat{k}^{2}
$$

and $\boldsymbol{\kappa}$ is the solution of

$$
\hat{\kappa} J_{n}^{\prime}(\hat{\kappa})=-i \beta_{e} \omega J_{n}(\hat{\kappa}) \text {. }
$$

Here, $\beta_{e}$ is the effective admittance at the wall; it is given by ${ }^{10}$

$$
\beta_{e}=(1 / 2) \delta(1-i) \omega^{-1}\left[\hat{k}^{2}+n^{2}+(\gamma-1) \omega^{2} / \sqrt{\mathrm{Pr}}\right],
$$

where $\operatorname{Pr}$ is the Prandtl number and $\delta=\sqrt{2 / \omega \mathrm{Re}}$ with the Reynolds number Re being based on the undisturbed speed of sound.

For small $\beta_{e}$, Eq. 74 has the approximate solution

$$
\hat{\kappa} \approx \kappa-i \beta_{e} \omega \kappa\left(n^{2}-\kappa^{2}\right)^{-1},
$$

where $J_{\hat{n}}^{\prime}(\kappa)=0$. Substituting for $\hat{\kappa}$ into Eq. 73 and solving for $\hat{k}$ gives

$$
\hat{k} \approx k+(1+i) \alpha,
$$

where

$$
\begin{aligned}
\alpha= & (1 / 2) \delta\left(\omega^{2}-k^{2}\right) k^{-1}\left(n^{2}+k^{2}-\omega^{2}\right)^{-1} \\
& \times\left[k^{2}+n^{2}+(\gamma-1) \omega^{2} / \sqrt{\operatorname{Pr}}\right] .
\end{aligned}
$$

Therefore, Eq. 72 can be rewritten in the form

$$
\phi(r, \theta, x, t) \approx A_{n}(x) J_{n}(\kappa r) \exp [i(k x-\omega t+n \theta)]+c c,
$$

where

$$
d A_{n} / d x=-(1-i) \alpha A_{n} .
$$

Since $\alpha$ is small, we can account for the effects of the acoustic boundary layer by modifying Eq. 49 in such a way that the resulting equation reduces to Eq. 80 for linear monochromatic waves; that is,

$$
\frac{\partial A}{\partial x}+k^{\prime} \frac{\partial A}{\partial t}+\frac{1}{2} i k^{\prime \prime} \frac{\partial^{2} A}{\partial t^{2}}=-(1-i) \alpha A-\frac{1}{2} i \epsilon^{2} \frac{\Lambda}{k} A^{2} \bar{A} .
$$

Letting $\partial A / \partial t=\partial^{2} A / \partial t^{2}=0$ and neglecting the nonlinear term in Eq. 81, one arrives at Eq. 80.

\section{ACKNOWLEDGMENT}

This work was supported in part by the NASA Langley Research Center under Grant NGR 47-004-109.

\section{APPENDIX A}

$$
\begin{aligned}
F(r)= & \kappa^{2} J_{m}(\kappa r) J_{m}^{\prime 2}(\kappa r)\left[\frac{1}{2}(\gamma-1) \omega^{2}+\frac{m(5 m-3)}{r^{2}}-3 \kappa^{2}+2 k^{2}\right]-\kappa J_{m}^{2}(\kappa r) J_{m}^{\prime}(\kappa r)\left[\frac{m^{2}}{r^{3}}+2 i \omega\left(\frac{2 m \Gamma_{1}}{r}+\Gamma_{2} \kappa r\right)\right] \\
& +\frac{3 \kappa^{2}}{r} J_{m}^{\prime 2}(\kappa r) J_{m+1}(\kappa r)-2 i \omega \kappa J_{m}^{\prime}(\kappa r)\left[-\Gamma_{2} \kappa r J_{m+1}^{2}(\kappa r)-2 \Gamma_{1} \kappa J_{m}(\kappa r) J_{m+1}(\kappa r)+\frac{2 m}{r} \Gamma_{3} J_{2 m}(2 \kappa r)-2 \Gamma_{3} \kappa J_{2 m+1}(2 \kappa r)\right] \\
& -\left[(\gamma-1) \omega^{2}+\frac{2 m^{2}}{r^{2}}+2 k^{2}\right]\left\{J_{m}^{3}(\kappa r)\left[\frac{m^{2}}{2 \gamma^{2}}+\frac{1}{2} k^{2}+(\gamma-1) \omega^{2}+2 i \omega \Gamma_{1}\right]+2 i \omega J_{m}(\kappa r)\left[\Gamma_{2} \gamma J_{m}(\kappa r) J_{m+1}(\kappa r)+\Gamma_{3} J_{2 m}(2 \kappa r)\right]\right\} .
\end{aligned}
$$

${ }^{1}$ D. T. Blackstock, "Nonlinear Acoustics (Theoretical)," in American Institute of Physics Handbook, D. Bray, Ed. (McGraw-Hill, New York, 1972), Chap. 3n, pp. 3-183-3-205.

${ }^{2}$ A. H. Nayfeh, J. E. Kaiser, and D. P. Telionis, "The Acoustics of Aircraft Engine-Duct Systems," AIAA J. (to be published).

${ }^{3}$ A. B. Coppens, J. Acoust. Soc. Am. 49, 306-318 (1971).
${ }^{4}$ F. M. Pestorius and D. T. Blackstock, Interagency Symposium on University Research in Transportation Noise Proceedings (Stanford University, Stanford, CA, 1973), Vol. II, pp. 565-55.

${ }^{5}$ A. H. Nayfeh, Perturbation Methods (Wiley-Interscience, New York, 1973), Chap. 6, pp. 228-307.

${ }^{6}$ L. I. Schiff, Quantum Mechanics (McGraw-Hill, New York, 
1955), p. 20.

${ }^{7}$ A. H. Nayfeh and M. -S. Tsai, "Nonlinear Wave Propagation in Acoustically Lined Circular Ducts," J. Sound Vib. 35, 77-89 (1974).
${ }^{8} \mathrm{~J}$. B. Keller and M. H. Millman, J. Acoust. Soc. Am. 49, 329-333 (1971).

${ }^{9}$ J. B. Keller, J. Acoust. Soc. Am. 55, 524-527 (1974).

${ }^{10}$ A. H. Nayfeh, J. Acoust. Soc. Am. 54, 1737-1742 (1973). 